\title{
Comprehensive characterization of the cis-regulatory code responsible for the spatio-temporal expression of olSix3.2 in the developing medaka forebrain Ivan Conte and Paola Bovolenta
}

Address: Departamento de Neurobiología Celular, Molecular y del Desarrollo, Instituto Cajal, CSIC, Dr Arce, Madrid 28002, Spain.

Correspondence: Paola Bovolenta. Email: bovolenta@cajal.csic.es

Published: 6 July 2007

Genome Biology 2007, 8:RI37 (doi: I0.1 I86/gb-2007-8-7-r|37)

The electronic version of this article is the complete one and can be found online at http://genomebiology.com/2007/8/7/RI37
Received: 23 February 2007

Revised: 5 June 2007

Accepted: 6 July 2007

(C) 2007 Conte and Bovolenta.; licensee BioMed Central Ltd.

This is an open access article distributed under the terms of the Creative Commons Attribution License (http://creativecommons.org/licenses/by/2.0), which permits unrestricted use, distribution, and reproduction in any medium, provided the original work is properly cited.

\begin{abstract}
Background: Embryonic development is coordinated by sets of cis-regulatory elements that are collectively responsible for the precise spatio-temporal organization of regulatory gene networks. There is little information on how these elements, which are often associated with highly conserved noncoding sequences, are combined to generate precise gene expression patterns in vertebrates. To address this issue, we have focused on Six 3 , an important regulator of vertebrate forebrain development.

Results: Using computational analysis and exploiting the diversity of teleost genomes, we identified a cluster of highly conserved noncoding sequences surrounding the Six 3 gene. Transgenesis in medaka fish demonstrates that these sequences have enhancer, silencer, and silencer blocker activities that are differentially combined to control the entire distribution of Six3.

Conclusion: This report provides the first example of the precise regulatory code necessary for the expression of a vertebrate gene, and offers a unique framework for defining the interplay of trans-acting factors that control the evolutionary conserved use of Six 3 .
\end{abstract}

\section{Background}

Embryonic development is coordinated by networks of evolutionary conserved regulatory genes that encode transcription factors and components of cell signaling pathways, which in many instances are repetitively exploited in space and time to generate appropriate outcomes in target cells.

Progressive specification of the vertebrate prosencephalon indeed follows this rule $[1,2]$ and requires, among other factors, recurrent use of Six 3 , which is a member of the Six/sine oculis family of homeobox transcription factors [3]. In all vertebrates, Six 3 is expressed from the neurula stage in the ante- riormost neural plate and then in its derivatives: the developing eyes and olfactory placodes, the hypothalamic pituitary regions, and the ventral telencephalon. In mouse and chick, this distribution overlaps with that of its closely related homolog, namely Six6 [3]. However, with time Six3 and Six6 expressions progressively segregate to different brain regions, and Six 3 - but not Six6 - is additionally expressed in the olfactory bulb, cerebral cortex, hippocampus, midbrain, and cerebellum [4]. Consistent with this expression, Six3-null mice die at birth, lacking most of the head structures anterior to the midbrain, including eyes [5], and mutations in $S_{I} X_{3}$ have been found in humans affected 
by holoprosencephaly and aprosencephaly/atelencephaly $[6,7]$. During mammalian lens induction, Six 3 is essential in the presumptive lens ectoderm to activate Pax6 and possibly Sox2 expression [8]. In addition, morpholino-based knockdown of the medaka fish Six3 demonstrates the concentration-dependent need for the function of this transcription factor for proximo-distal patterning of the optic vesicles [9]. Biochemical and functional studies have also shown that Six3, as well as Six6, can induce ectopic retinal tissues and control retinal neuroblast proliferation, acting as transcriptional repressors through the interaction with members of the groucho family of transcriptional co-repressors [10-15]. Furthermore, Six3, but not Six6, functionally interacts with the DNA replication inhibitor Geminin, controlling the balance between cell proliferation and differentiation with a mechanism that is independent of transcriptional regulation [16].

How the activity of Six 3 - or that of any other gene with multiple functions during embryo development - is diversified remains to be elucidated. This could be facilitated by defining the precise gene regulatory network that controls its spatiotemporal expression. It is now well established that control of gene expression is executed through sets of cis-regulatory regions within the noncoding DNA of animal genomes. These cis-regulatory modules have variable length and contain clusters of DNA-binding sites for different transcription factors. These modules work as promoter enhancers or silencers and collectively constitute a unique code for the switching on and off of gene activity [17-19].

The experimental definition of the organization of these specific cis-regulatory elements has progressed substantially in both Drosophila and sea urchin [17]. In contrast, our understanding of how these modules are combined to generate precise gene expression patterns in vertebrates is still rather limited. Possible causes of this are the increased genome complexity and the slow and laborious process of testing the functional significance of identified elements in mammals [20]. Recently, however, computational approaches based on multispecies genomic sequence alignments, combining both closely related and highly divergent organisms, have facilitated identification of highly conserved noncoding sequences, which in many cases appear to coincide with the regulatory modules of genes that play critical roles in development. Analyses of the complex regulation of genes such as Sox2, Sox9, Otx2, Shh, and Irx provide some illustrative examples [21-27]. Functional testing of 'enhancer' activity has also progressed, thanks to the use of alternative and relatively faster 'transgenic' approaches based on the use of nonmammalian vertebrate model systems $[20,25]$.

Here, we have taken advantage of both the power of computational analysis and the particular compact genome and high transgenesis efficiency of the medaka fish (Oryzia latipes) [28] to dissect the regulatory control of one of the two Six 3 medaka homologs, olSix3.2, that we identified during the course of this study. olSix3.2 is more closely related to the mammalian Six3 than the previously described medaka homolog [29] (hereafter referred to as 'olSix3.1'). Similar to other related studies [23-25], we identified and functionally characterized sets of cis-regulatory modules that control the olSix3.2 promoter, showing that at least some of these cisregulatory elements are conserved in other vertebrates, although they are dispersed over a greater stretch of DNA. Going a step further, we have also used combinations and deletions of the identified cis-regulatory modules to elucidate the regulatory code of olSix3.2, which is composed of two enhancers, two silencers, and two 'silencer blockers' used in a combinatorial manner. This comprehensive description of the olSix3.2 cis-regulatory code provides a unique framework for defining the network of trans-acting factors that control the evolutionary conserved activity of Six 3 during forebrain development.

\section{Results}

Isolation, characterization, and expression of olSix3.2

In order to identify the elements that regulate Six 3 expression using the medaka fish (Oryzia latipes) as a model, we used the available olSix3.1 coding sequence (AJoo0937) as a query to search public databases (see Materials and methods, below) for the ortholog genomic loci of the closely related species Fugu rubripes, Tetraodon nigroviridis, and Danio rerio (zebrafish). This search retrieved four different loci, one for the fugu and the tetraodon, and two for the zebrafish (six $3 a$ and $\left.\operatorname{six}_{3} b\right)$. Alignment of about 20 kilobases $(\mathrm{kb})$ of the retrieved sequences upstream of the Six 3 translational start sites identified a cluster of conserved noncoding blocks roughly contained within the first $4.5 \mathrm{~kb}$ (data not shown). In the case of the zebrafish, alignment of the six $3 a$ or six $3 b$ loci yielded comparable results. This information was used to amplify from genomic DNA a fragment of the medaka Six3 locus that contains the corresponding conserved noncoding blocks and the entire first exon.

Interestingly, nucleotide and amino acid sequence alignment of the partially amplified olSix3 coding region did not completely overlap with that reported for the previously identified olSix3.1 [29] but identified - as in zebrafish and Xenopus [30,31] - a second Six3-related gene in the medaka genome, namely olSix3.2 (AM494407).

Cloning and sequencing of the entire olSix3.2 coding region revealed a two-exon structure, similar to that of olSix 3.1 and the mouse Six3, in which the first exon encodes the Six and homeobox domains. olSix3.1 and olSix3.2 exhibited $76 \%$ and $63 \%$ identity at the nucleotide and amino acid levels, respectively. Interestingly, comparison of the amino acid sequence ( $81 \%$ versus 59\%; Additional data file 1 ) and genomic organization, together with phylogenetic analysis (Additional data file 2), demonstrated that olSix3.2 was more closely related to the mammalian Six3 than the previously identified olSix3.1, 
which instead falls in between the Six 3 and Six6 branches of the family (Additional data file 2).

olSix 3.1 is expressed in the anterior embryonic shield and the developing eye [29]. To determine whether the newly identified gene and the initially identified homolog had similar distributions, we compared the expression domain of olSix3.2 with those of olSix3.1 and the related olSix6 [13] using wholemount in situ hybridization. As for olSix 3.1, olSix 3.2 was first detected in the anterior neural plate at late gastrula stages but was additionally expressed in the anterior axial mesoderm at St16 (Figure 1a-c). At the optic vesicle stage, both olSix3.2 and olSix3.1, but not olSix6, were expressed in the forebrain. However, although olSix3.2 was more abundant in the presumptive telencephalon (Figure 1e,h), olSix3.1 was predominant in the optic area (Figure 1d,g). This distribution was more evident at later stages of development, when both olSix3.1 and olSix6, which first appears at the optic cup stage (Figure 1l) [13]), were strongly expressed in the developing neural retina, optic stalk, and preoptic and hypothalamic areas (Figure 1j,1,m,o,p,r). In contrast, olSix3.2 mRNA was distributed in the developing lens, olfactory pits, telencephalon, neural retina, anterior hypothalamus, and anterior and posterior thalamus (Figure 1k,n,q). During retinal neurogenesis, olSix3.1 was mostly confined to the inner nuclear layer (Figure 1s), and olSix3.2 and olSix6 to the retinal ganglion and amacrine cells (Figure 1t,u).

In conclusion, the distribution of olSix 3.2 appeared closely related to that reported for the chick and mouse Six3 $[4,32,33]$, whereas the combined expression patterns of olSix3.1 and olSix6 resembled that reported for Six6 [34,35].

\section{The cis-regulatory elements responsible for olSix3.2 expression are contained in a $4.5 \mathrm{~kb}$ genomic region ending with a distal 'silencer'}

On the basis of this expression pattern, we next searched for the elements that could be involved in the regulation of olSix3.2 expression. Alignment of the amplified olSix3.2 genomic sequence with the corresponding sequences from fugu, tetraodon, and zebrafish (analyses involving sixza and $s i x 3 b$ yielded similar results) identified ten conserved noncoding blocks within the $4.5 \mathrm{~kb}$ upstream of the translational start site olSix3.2 (Figure 2a).

Owing to selective pressure, functional elements in genomes evolve at a slower pace than nonfunctional regions [36-39]. A number of recent studies have functionally demonstrated that a proportion of the highly conserved noncoding regions present in vertebrate genomes correspond to regulatory elements with enhancer activity $[21,39]$. We therefore asked whether the region containing the cluster of ten highly conserved noncoding elements was necessary and sufficient to control the entire expression of olSix3.2.

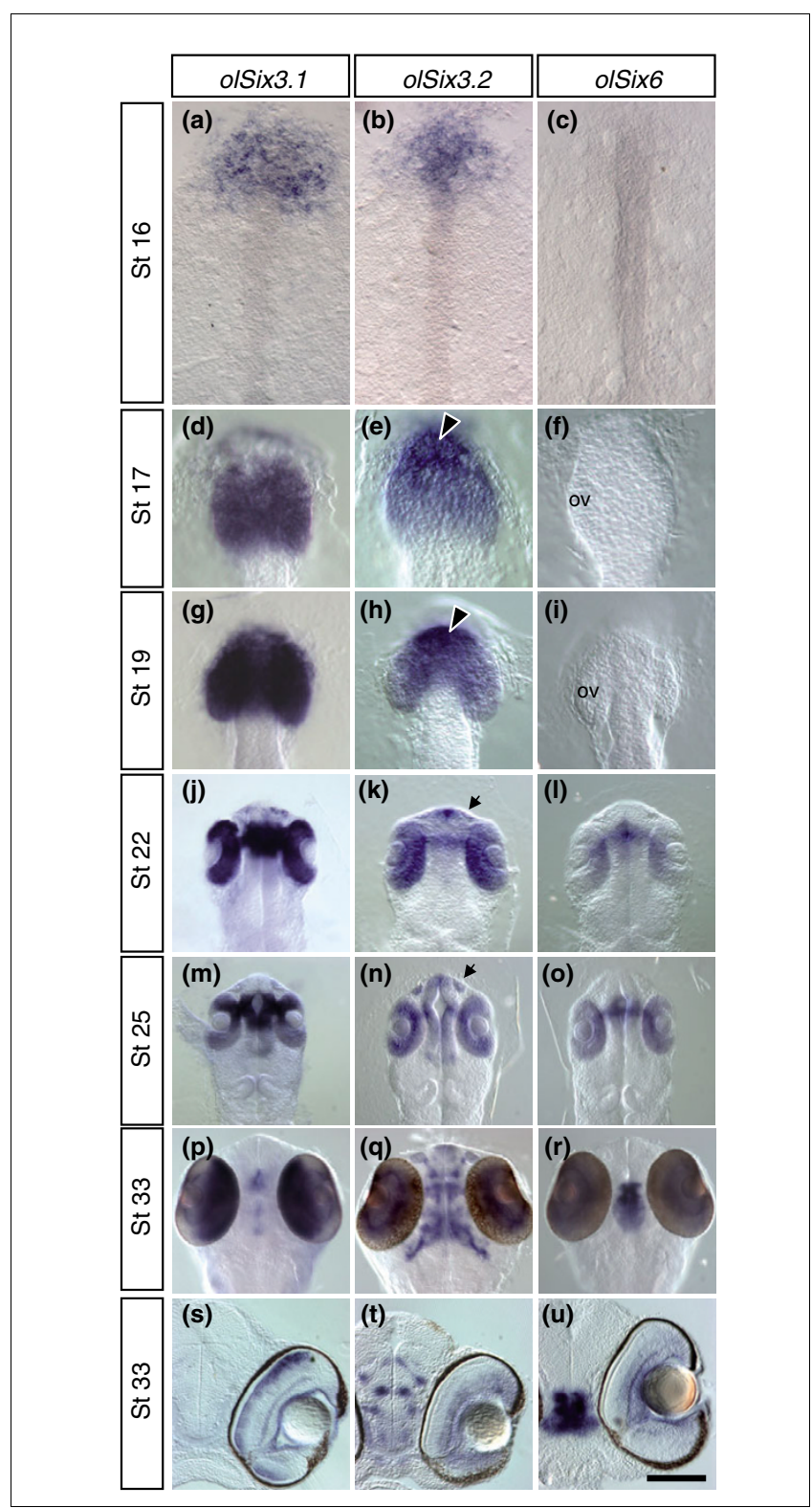

\section{Figure I}

Comparative analysis of olSix3.I, olSix3.2, and olSix6 expression pattern during embryonic development. Medaka embryos at different developmental stages (as indicated in the panels) were hybridized in toto with specific probes, as indicated on the top of each column. (a to $\mathbf{r}$ ) Anterior dorsal views; ( $\mathbf{s}$ to $\mathbf{u}$ ) frontal vibratome sections through the eye. From St 16 to St 19, only olSix3.I and olSix3.2 are expressed in the anterior neural plate (panels a to $c$ ) and then in the presumptive telencephalon and optic vesicles (panels $d$ to i), although olSix 3.1 is more abundant in the optic vesicles (panels $\mathrm{d}$ and $\mathrm{g}$ ) and olSix3.2 in the telencephalic region (arrowheads in panels e and h). From St22 onward, when olSix 6 mRNA also becomes detectable, the three genes are coexpressed, albeit at different levels, in the developing neural retina, optic stalk, and pre-optic and hypothalamic area (panels j to $r$ ). In addition, olSix3.2 is distributed in the developing lens, olfactory pits (panels $k$ and $n$; arrow), telencephalon, and anterior and posterior thalamus (panels $k, n$, and q). During retinal neurogenesis, olSix3.2 and olSix6 are restricted to the retinal ganglion and amacrine cells (panels $\mathrm{t}$ and $\mathrm{u}$ ), whereas olSix3.I is restricted to the inner nuclear layer (panel $s$ ). 
(a)

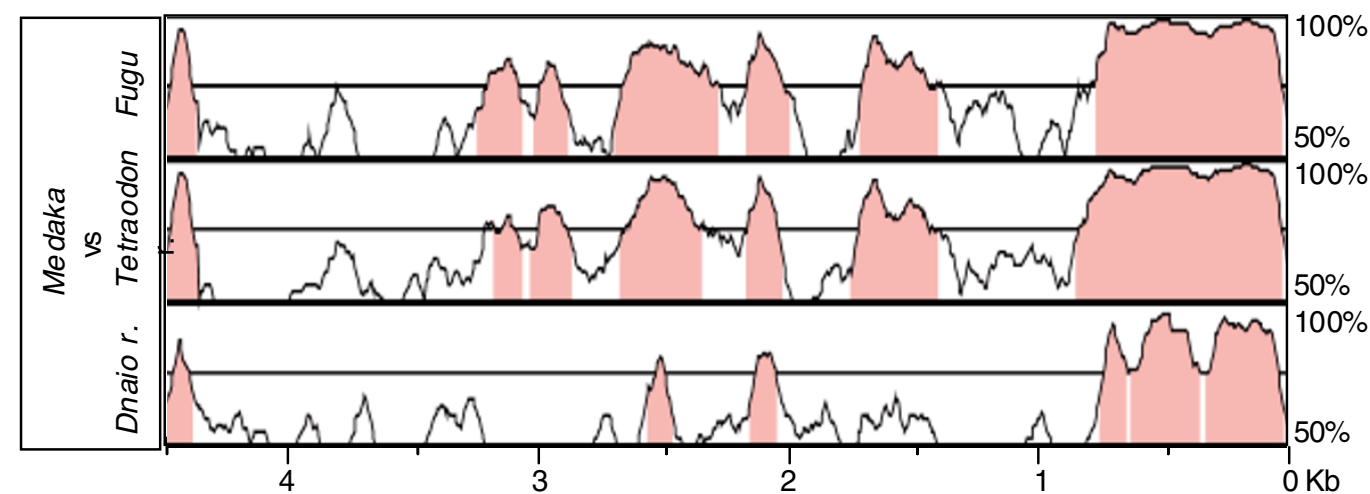

(b)

$\mathrm{cl}$

A B

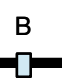

C
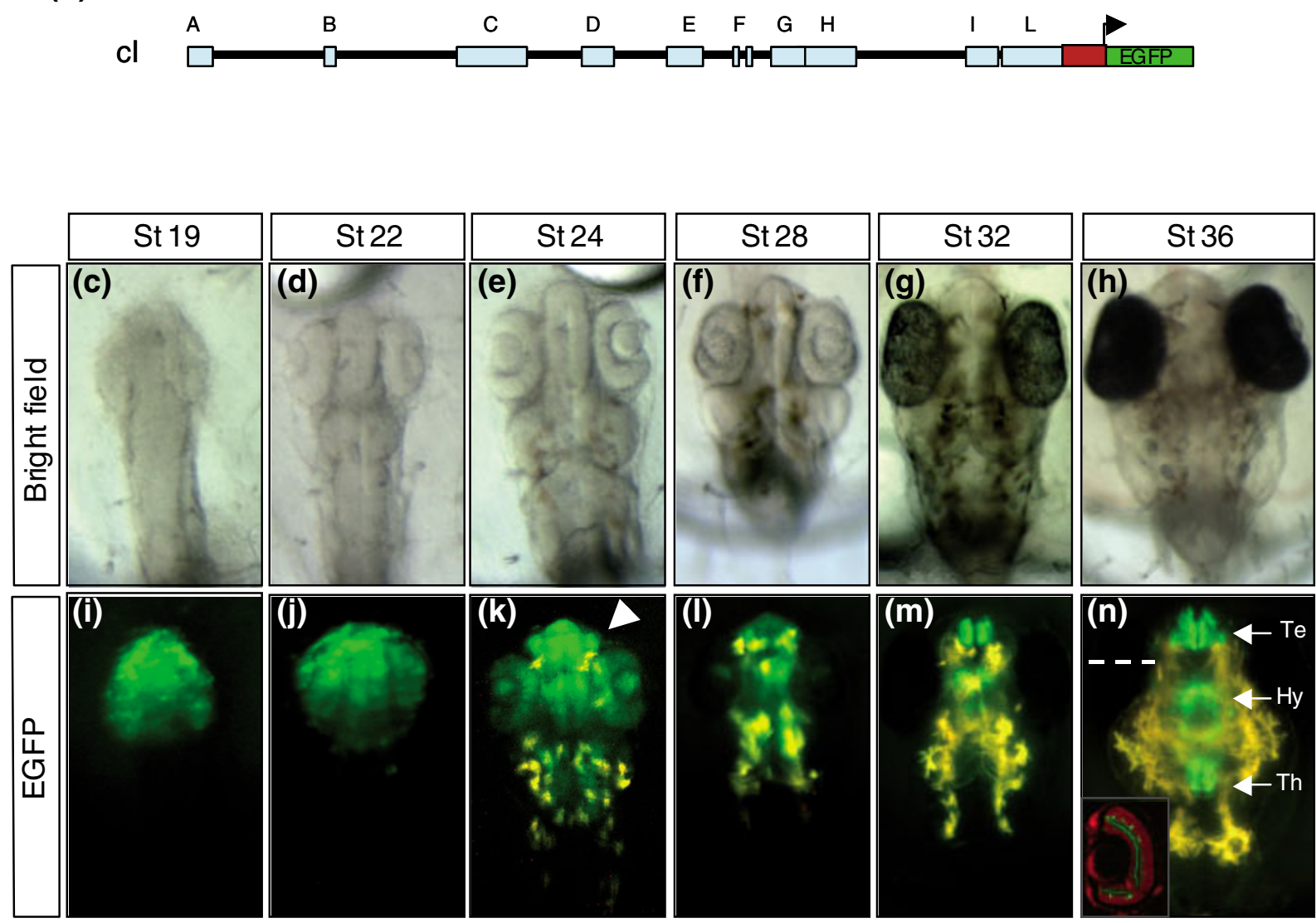

\section{Figure 2}

The cis-regulatory elements responsible for the olSix3.2 expression are contained in a $4.5 \mathrm{~kb}$ genomic region. (a) VISTA comparison of the $5^{\prime}$ olSix 3 genomic region plotted against those from Fugu rubripes, Tetraodon nigroviridis, and Danio rerio. The blocks of sequences ( $75 \%$ identity over 100 base pairs) conserved among the four species are indicated in pink. (b) Schematic structure of the $5^{\prime}$ olSix3.2 genomic region/enhanced green fluorescent protein (EGFP) reporter construct (cl) containing ten highly conserved noncoding regions represented as light blue rectangles $A$ to $L$. The red rectangle represents the 5'-untranslated region and the first nine nucleotides of the olSix3.2 coding sequence in frame with a nuclear EGFP reporter (green). (c to $\mathbf{h}$ ) Bright field images; and (i to $\mathbf{n}$ ) epi-fluorescence dorsal views of cl transgenic embryos at different stages of development (as indicated). Note that the $\mathrm{cl}$ construct drives EGFP reporter expression to the same olSix3.2 expression domain, recapitulating its entire pattern (compare with Figure I). The arrowhead in panel $k$ points to the olfactory pits. The inset in panel $n$ shows a frontal section through the eye (dotted line), where EGFP is expressed in the amacrine cells. The section was counter-stained with propidium iodine (red). Hy, hypothalamus; Te, telencephalon; Th, thalamus. 
(a)

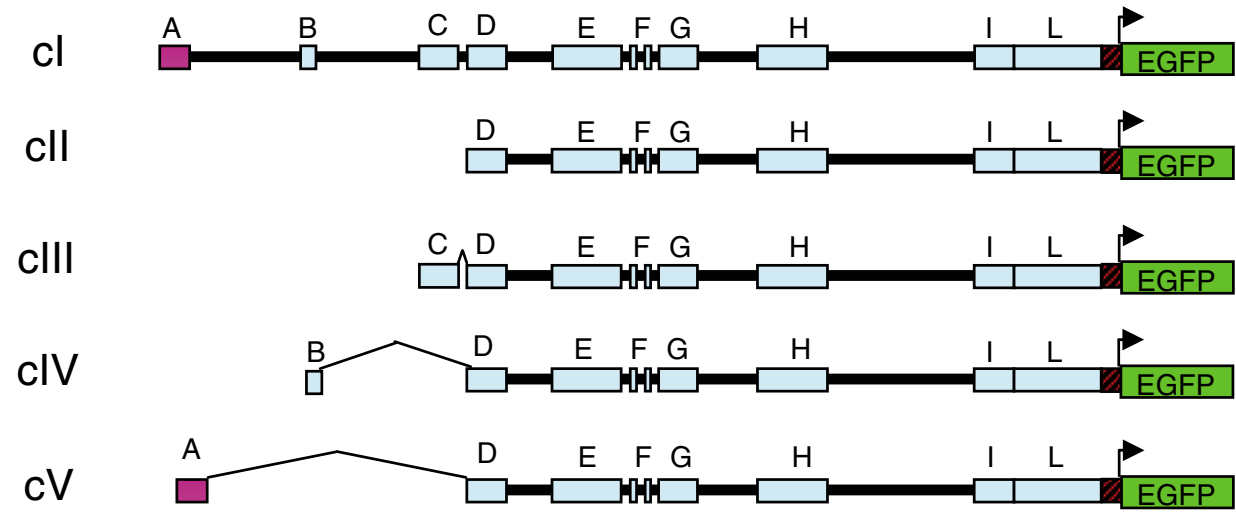

\begin{tabular}{|c|c|}
\hline NE & EPE \\
\hline+ & - \\
\hline+ & + \\
\hline+ & + \\
\hline+ & + \\
\hline+ & - \\
\hline
\end{tabular}

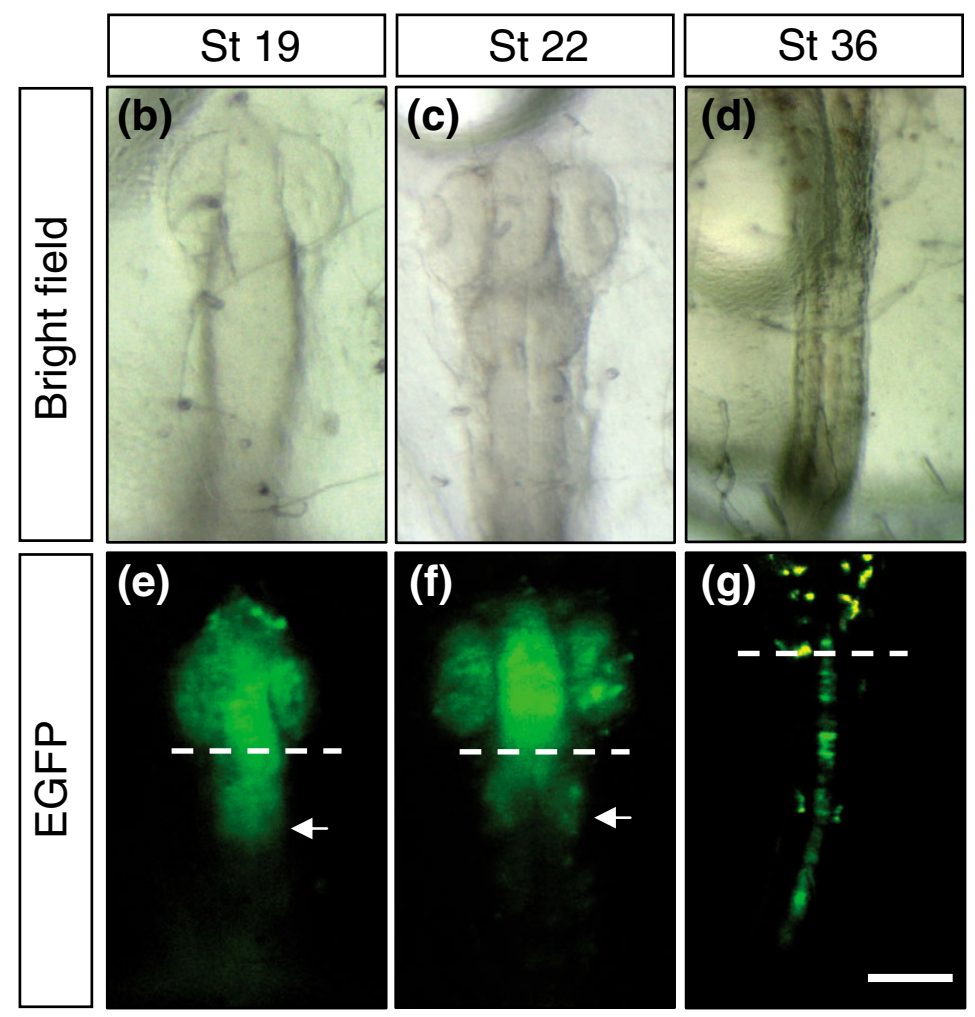

Figure 3

The most distal conserved module, A, is a silencer that restrains olSix 3.2 expression to the anterior neural plate. (a) Drawings to the left of the panel are schematic representations of the different constructs ( $c l$ to $\mathrm{cV}$ ) used to study the potential regulatory activity of modules $\mathrm{A}$ to $\mathrm{C}$, whereas the tables to the right summarizes the presence $(+)$ or absence $(-)$ of enhanced green fluorescent protein (EGFP) reporter expression observed with each construct and corresponding to the endogenous olSix3.2 expression domain (NE) or with an ectopic posterior expansion (EPE). The A module with silencer activity is depicted in purple. (b to d) Bright field images, and (e to g) epi-fluorescence dorsal views of cll transgenic embryos at different stages of development (as indicated). Note that the domain of EGFP expression is progressively expanded in the caudal direction (arrows in panels e and $f$ ), invading the spinal cord at St36 (panel g). Equivalent patterns were observed with the clll and clV transgenic lines. Dotted lines in panels e to $g$ indicate the caudal limit of endogenous olSix 3.2 expression. 
To this end we fused this $4.5 \mathrm{~kb}$ genomic region, including the first nine nucleotides of the coding sequence, in frame with a nuclear EGFP (enhanced green fluorescent protein) reporter (Figure 2b). This construct, containing the ten conserved noncoding blocks (termed A-L; Figure 2b), was used to generate three independent stable transgenic medaka lines, which all exhibited a spatio-temporal distribution of the reporter virtually identical to that observed for the endogenous olSix 3.2 both at embryonic (compare Figure 1 with Figure 2c-n) and adult stages (not shown). We thus concluded that this region was sufficient to control the entire expression of olSix3.2.

In addition to regulatory elements, sequence conservation could reflect the existence of natural anti-sense mRNAs [40] or of alternative and yet uncharacterized exons of Six3. However, reverse transcription polymerase chain reaction (RTPCR) analysis and in situ hybridization studies excluded these possibilities (data not shown). We thus assumed that the ten modules, identified on the basis of their conservation among teleosts (the precise nucleotide sequence of each module is provided in Additional data file 3), could all potentially contain elements that are involved in the regulation of olSix3.2. To test whether this assumption was correct, we generated a series of constructs (named cI to cXXVII) carrying different combinations of the A-L modules, which were then functionally assayed by generating and analyzing three independent stable transgenic lines for the vast majority of the constructs. In each case, the pattern of expression of the EGFP reporter was compared with that observed with construct I (cI), containing the full $4.5 \mathrm{~kb}$ sequence (Figure 2i-n) and was always consistent with that observed in $\mathrm{F}_{\mathrm{o}}$ injected embryos.

Embryos of a transgenic line carrying a construct in which the A to $\mathrm{C}$ modules had been deleted (cII; Figure 3a) showed a pattern of EGFP expression in the anteriormost neural tube similar to that observed with cI. However, embryos consistently exhibited an additional transient expansion of EGFP distribution to posterior mesencephalic regions (compare Figure 3e,f with Figure 2i,j and Figure 1h,k), which disappeared after St22. EGFP fluorescence was also consistently observed in the spinal cord starting from St34 (Figure 3d,g) up to adult stages. These observations suggested that, presumably, blocks D to L were sufficient to control normal olSix 3.2 expression, whereas the A to $\mathrm{C}$ modules contained a silencer(s), the activity of which was necessary to restrain olsix 3.2 expression to anterior domains of the neural tube throughout development. To determine the location of the silencer activity, we generated and functionally analyzed three different constructs containing the $\mathrm{D}$ to $\mathrm{L}$ modules in combination with the A, B, or C block (cIII to cV; Figure 3a). Only the presence of 134 base pairs (bp) of the A module could repress the posterior EGFP expansion, restoring the normal olSix3.2 distribution, which clearly identified the presence of a cis-regulatory silencer(s) in this sequence. In spite of sequence conservation, the $\mathrm{B}$ and $\mathrm{C}$ blocks instead did not appear to contribute to the spatio-temporal control of olSix3.2, at least in the context that we tested.

\section{Early expression of olSix3.2 in the anterior neural structures depends on one enhancer, whereas that in the lens placode requires the additional activity of four cis-regulatory modules}

We then sought to determine the functional relevance of the remaining $\mathrm{D}$ to $\mathrm{L}$ conserved modules. To this end we generated a series of additional constructs (named cVI to cXXII; Figure 4a) based on selective deletion of one or more modules at the time or by including different combinations of a few of them. Transgenesis analysis of these constructs demonstrated that the D module was necessary (cVI to cXVII; Figure 4a,c) and sufficient (cXIX; Figure 4a,e) to drive EGFP expression in all of the anterior neural structures from St16 to St23. In contrast, the $\mathrm{D}$ module was necessary but not sufficient (cXIX; Figure 4e) to control EGFP expression in the lens placode/lens vesicle, as normally observed for the endogenous olSix3.2 (Figure 4b). Indeed, the activity of modules E to $\mathrm{H}$ was further required for EGFP expression in the lens (cVI and cXVIII; compare Figure 4d with Figure 4e), because deletion of either one of them was sufficient to abrogate the reporter expression in the lens ectoderm (cXIX to cXXII; Figure 4a,e), suggesting that multiple cis-regulatory sequences spread along these four modules contribute to olSix 3.2 expression in this tissue. This is somewhat in contrast with the apparently simpler regulation of olSix 3.2 distribution in the early neural tissue, which mostly depends on the D block.

\footnotetext{
Figure 4 (see following page)

Different constructs used to generate stable transgenic lines and corresponding distribution of EGFP reporter in expected olSix3.2 expression domains. (a) Drawings to the left of the panel are schematic representations of the different constructs (cl and $\mathrm{cVI}$ to $\mathrm{cXXII)}$ used to generate stable transgenic lines, whereas the tables to the right summarize the presence $(+)$ or absence $(-)$ of enhanced green fluorescent protein (EGFP) reporter expression corresponding to the expected olSix3.2 expression domain at different stages of differentiation, in the retina or ectopically in the spinal cord. The red box represents the 5'-untranslated region and the first nine nucleotides of the olSix3.2 coding sequence, in frame with a nuclear EGFP reporter, whereas the dark blue box represents the minimal tyrosine kinase promoter. (b to e) The images show frontal vibratome sections through the optic cup of in situ hybridized (b) wild type and (c) cVII, (d) cXVIII and (e) cXIX transgenic lines. Note that module D alone is sufficient to drive EGFP expression in the hypothalamus and neural retina but not in the lens (empty arrow in panel e), whereas in its absence EGFP expression is completely lost (panel b). A similar absence of EGFP expression was observed in the $\mathrm{CVIII}$ to $\mathrm{CXVII}$ transgenic lines, all of which lack module $\mathrm{D}$. Note also that the combination of modules $\mathrm{D}$ to $\mathrm{H}$ is necessary for expression in the lens placode (arrow in panel d), as indicated by in situ hybridization of the endogenous olSix3.2 distribution (arrow in panel b). Hy, hypothalamus; NR, neural retina.
} 

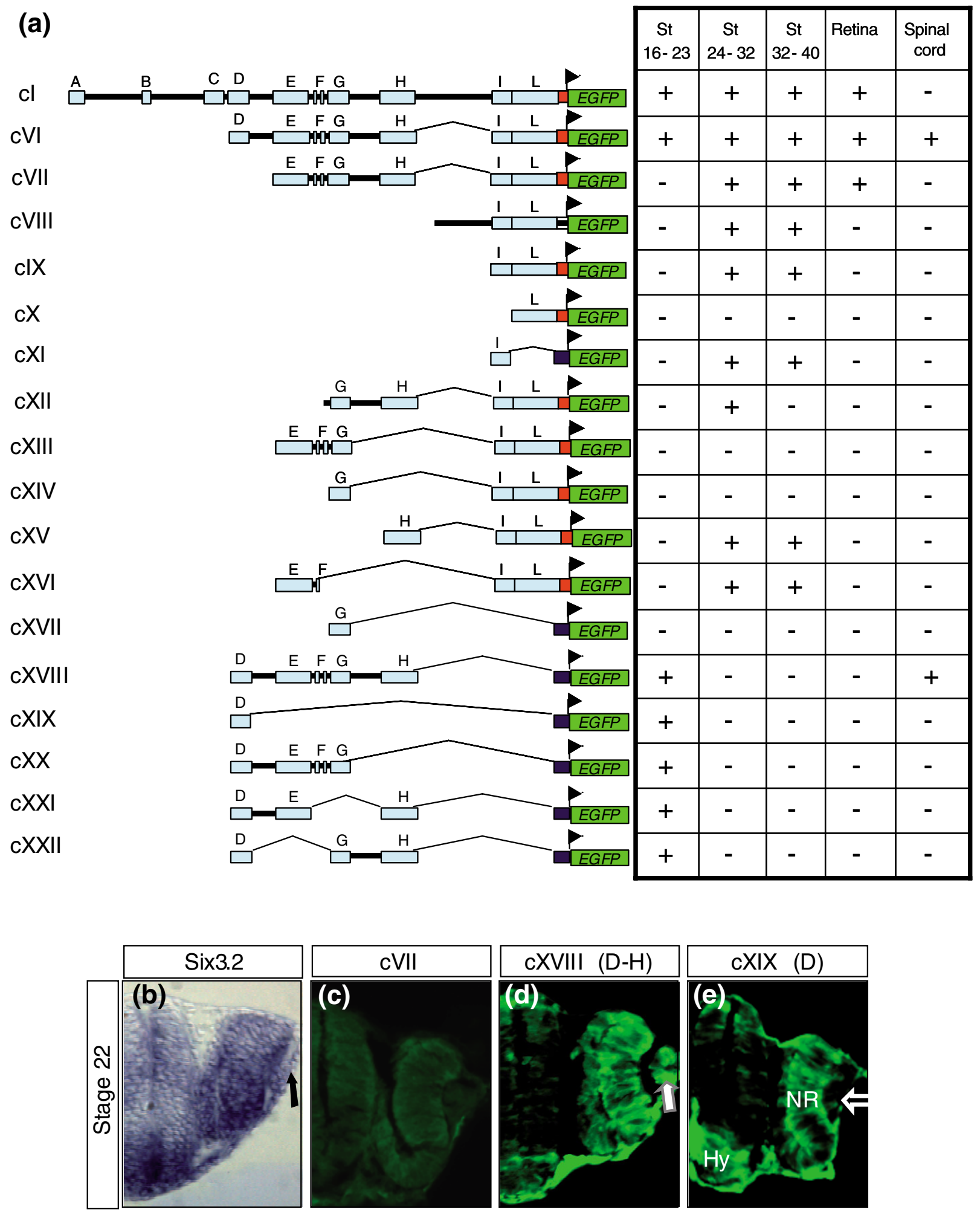

Figure 4 (see legend on previous page) 
Notably, modules D to H (cXVIII; Figure 4a) were also sufficient to induce caudal expansion of reporter expression, with a pattern identical to that observed in the absence of the A module (Figure $3 \mathrm{e}-\mathrm{g}$ ), indicating that modules I and $\mathrm{L}$ do not contribute to this expansion or to early expression of the gene.

\section{During organogenesis, appropriate expression of olSix3.2 requires the combined activity of two silencers, one enhancer, and two putative 'silencer blockers'}

To determine whether these last two modules were functionally relevant to any other aspect of olSix 3.2 expression, we designed a number of constructs in which modules I and L were assayed separately (cX and cXI), in conjunction (cIX), and combined with the olSix3.2 endogenous promoter (cX) or with the minimal tyrosine kinase promoter (cXI). Injections of cX were not associated with EGFP expression in any region of the embryo at any stage (Figure 4a). This indicates that, as in the case of modules B and C, the L block had no enhancer silencer activity relevant to the regulation of olSix3.2, at least in the tested conditions, although its sequence is strongly conserved among all vertebrates. In contrast, the activity of block I was clearly linked to control of olSix 3.2 distribution in the forebrain starting from St26 onward, when EGFP was gradually observed, with progressively increasing intensity, first in the telencephalic, then in the hypothalamic, and finally in the thalamic region (Figures $4 \mathrm{a}$ and $5 \mathrm{c}$ ). This recapitulates the endogenous expression of the gene (Figure 1q).

To determine the minimal region of module I involved in the control of this expression, we engineered five 5 ' to 3 ' stepwise deletions covering the entire module (cXXIII to cXXVII; Figure 5a). Notably, deletions two, three, and four resulted in progressive abrogation of EGFP expression in the thalamic, hypothalamic (Figyre 5b-d), and telencephalic regions (not shown). This strongly suggests that module I contains a 5 ' to 3 ' organized succession of cis-regulatory elements that control the posterior to anterior spatio-temporal organization of olSix3.2 expression in the developing brain. This interpretation was further supported by the injection of two internal deletion constructs (cXXVIII and cXXIX) in which the stretches of nucleotides apparently responsible for hypothalamic and telencephalic expression were removed from cXXIII (Figure 5a). Indeed, in 11\% (close to transgenic efficiency) of the embryos analyzed in $\mathrm{F}_{\mathrm{o}}$, EGFP fluorescence was not detected in the telencephalon (cXXVIII; Figure $5 \mathrm{f}$ ) or in the hypothalamus and telencephalon (cXXIX; Figure 5g), clearly indicating that deleted elements are the main driver of olSix3.2 expression in these regions.

The elements contained in the I module appeared to suffice in terms of regulating late olSix3.2 embryonic expression in the brain. Nevertheless, we considered whether any additional module could modify their activity. Transgenic embryos carrying cXIV, in which the G module was combined with the I module, had no reporter expression in the brain (Figure 4a), raising the possibility that the $\mathrm{G}$ module contained a 'silencer' that, in turn, could be normally regulated by a 'silencer blocker', as previously proposed [41,42]. Addition of the $\mathrm{H}$ block (cXII) proved that this was the case, because its presence restored reporter expression, although only from St26 to St32. Further addition of the E block (cVII, containing E, G, $\mathrm{H}$ and I) appeared to overcome the effect of the $\mathrm{G}$ silencer from St32 onward. Thus, proper regulation of late olSix3.2 embryonic expression requires the participation of five different modules - one enhancer, one silencer, and two silencer blockers - in addition to the silencer activity contained in the distal A module (Figure 6c,d).

When tested alone, block I did not drive EGFP expression in the differentiating retina, whereas activity of the D block was sufficient to maintain reporter expression only in the prospective neural retina (Figure 4a,d,e). Thus, olSix3.2 expression in the differentiating retina appeared to depend on a combination of modules different from those tested thus far. The search for this code demonstrated that only the combined activity of the E to I modules (cVII; Figure 4a) was effective in supporting EGFP expression in the late developing retina.

\section{Identification and characterization of conserved regions among vertebrate}

Altogether these data provide a detailed picture of the regulatory code that governs olSix 3.2 expression during eye and brain development in medaka. As summarized in Figure 6, this spatio-temporal code is provided by the combined use of at least seven different modules, all conserved among fishes, with distinct enhancer, silencer, or silencer blocker activities. The next logical question was whether this regulatory organisation was conserved in the Six 3 locus of vertebrates other than fishes.

To address this problem, we used the characterized olSix 3.2 regulatory region as a query to search public databases

\footnotetext{
Figure 5 (see following page)

Module I contains a 5' to $3^{\prime}$ organized sequence of cis-regulatory elements that control the posterior to anterior expression of olSix3.2 in brain. (a) The drawings illustrate the design of the $c X X I I I$ to $c X X I X$ constructs use to determine the arrangement of the cis-regulatory elements within module I, using five progressive deletions of about 50 base pairs, indicated by a gradient of blue colors. (b) Nucleotide sequence of module I, in which the precise position of the deletions is indicated with the same gradient of blue colors. (c to g) Epi-fluorescence dorsal views of cXXIV to cXXIX transgenic embryos that show the loss of thalamic (panel $d$ ), hypothalamic (panels e and g), and telencephalic (panels $f$ and g) reporter expression. cXXVII transgenic embryos exhibited no enhanced green fluorescent protein (EGFP) expression. Hy, hypothalamus; Te, telencephalon; Th, thalamus.
} 
(a)

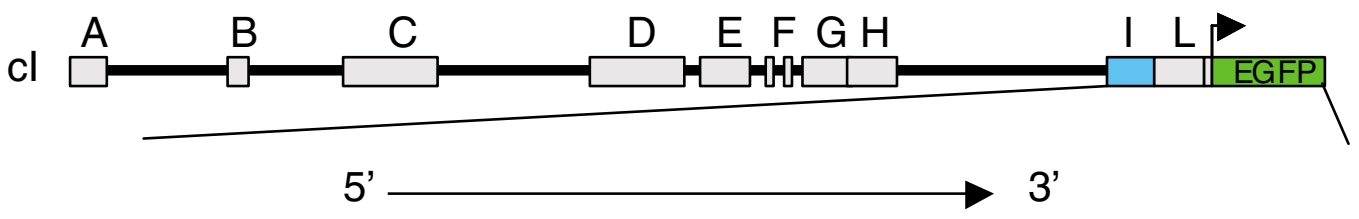

cXXIII

cXXIV

cXXV

cXXVI

cXXVII

CXXVIII

CXXIX

\begin{tabular}{|c|c|c|c|c|}
\hline & Th & $\mathrm{Hy}$ & $\mathrm{Te}$ & TEGFP \\
\hline & Th & $\mathrm{Hy}$ & $\mathrm{Te}$ & $=P$ \\
\hline & & $\mathrm{Hy}$ & $\mathrm{Te}$ & EGFP \\
\hline & & & $\mathrm{Te}$ & EGFP \\
\hline & & & & TEGFP \\
\hline & Th & $\mathrm{Hy}$ & & 化 \\
\hline & Th & & & 化 \\
\hline
\end{tabular}

(b)

CTTCGCTATAGGGAAATCTGCATGGAAATAATGTGCAGATTGACTTGCTTCCATTCAAAATTCCC
GAAGCGATATCCCTTTAGACGTACCTTTATTACACGTCTAACTGAACGAAGGTAAGTTTTAAGGG

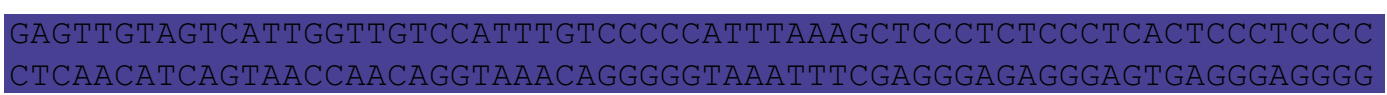

GTCTCTACTAAGCATCTCCAGTCTACATATCTTCTTTAGCTTTAACGAGCCTCGTTAAGATCGCA CAGAGATGATTCGTAGAGGTCAGATGTATAGAAGAAATCGAAATTGCTCGGAGCAATTCTAGCGT

AтAАTAтTCCACCCTCTAATTGCTCATTCCATTCAGCAGATAGGCGAGCATTGGCTTGTGCCTGA TATTATAAGGTGGGAGATTAACGAGTAAGGTAAGTCGTCTATCCGCTCGTAACCGAACACGGACT

TGCGCGCGGTGCGGTGGGAGGGTTGCTGTGGAGATCCTAGACTCTGATAACCCCCCGTGCGTGCT ACGCGCGCCACGCCACCCTCCCAACGACACCTCTAGGATCTGAGACTATTGGGGGGCACGCACGA

GCACAAGTGGTGAAAGCCTCGCGCTACGTACTGGCTAATGATTGGCACGCTTGACAGTGATTGGC CACGACGTGTTCACCACTTTCGGAGCGCGATGCATGACCGATTACTAACCGTGCGAACTGTCACT

AGGGCTGCCATGACAACGCTACAACGACACCAAGAAGACCAATAGAAAAGGGAAACAAAATGTTT TCCCGACGGTACTGTTGCGATGTTGCTGTGGTTCTTCTGGTTATCTTTTCCCTTTGTTTTACAAA

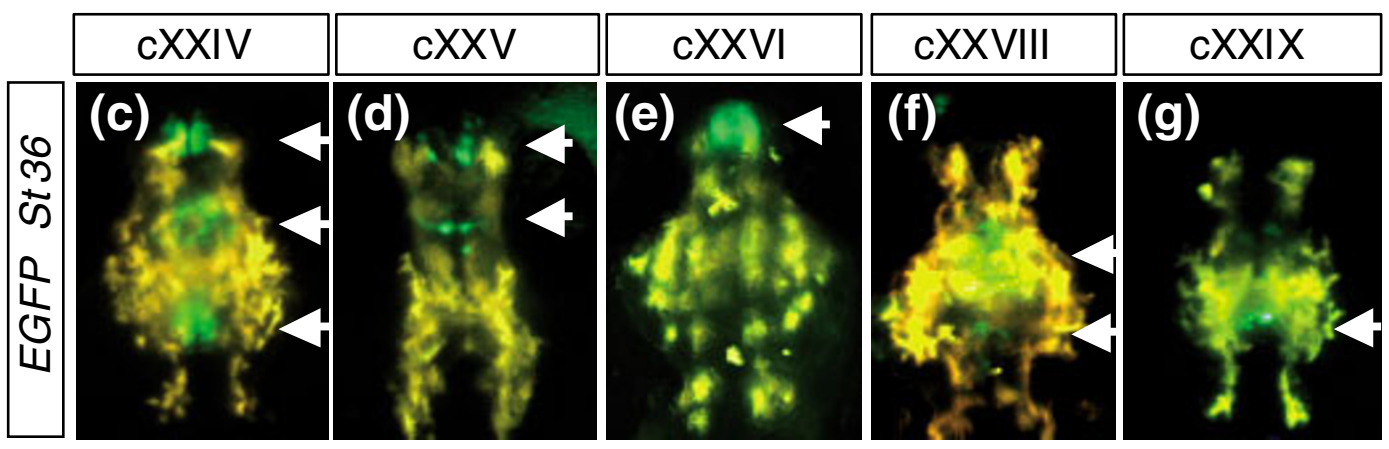

Figure 5 (see legend on previous page) 


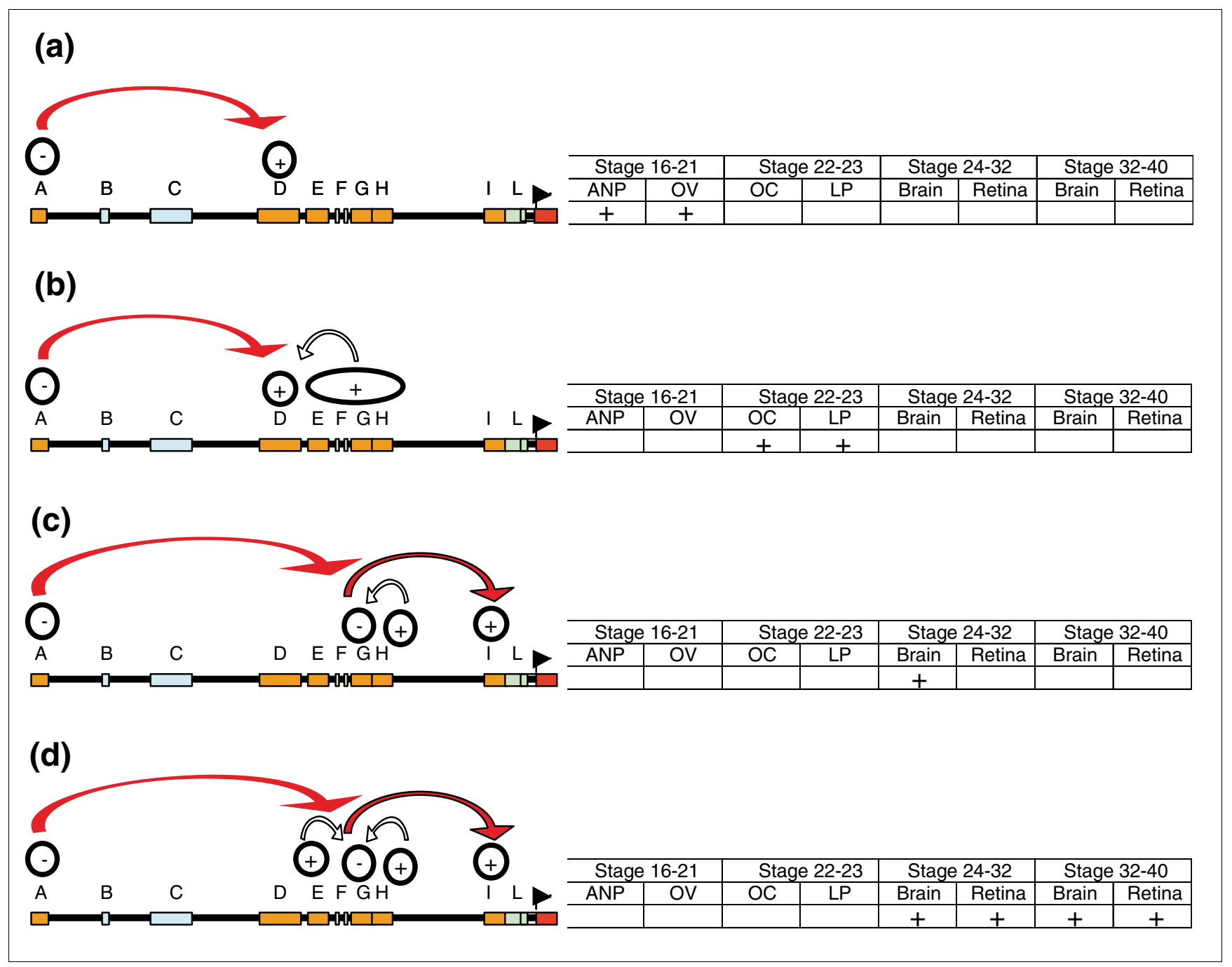

Figure 6

Summary of the regulatory code that control the entire expression of olSix3.2. (a) Early expression of olSix3.2 in the forebrain and eye depends on enhancers in module $D$ and a silencer activity (activities) in module A. (b) olSix3.2 expression in the lens placode requires multiple elements distributed along modules $\mathrm{D}$ to $\mathrm{H}$. (c) During organogenesis, correct olSix3.2 expression requires the activity of different enhancer arranged in a $5^{\prime}$ to 3 ' mode within module $\mathrm{I}$. The activity of $\mathrm{I}$ is repressed by module $\mathrm{G}$, which, in turn, is neutralized initially by module $\mathrm{H}$ and at later stages (d) by the combined activity of the $\mathrm{E}$ and $\mathrm{H}$ silencers. Module $\mathrm{A}$ is necessary at all stages analyzed to prevent reporter expansion to caudal central nervous system.

(Genome Bioinformatics UCSC [University of California, Santa Cruz]) for the ortholog regions in vertebrates other than fishes. This analysis showed that only part of the modules identified in teleosts were conserved among all vertebrate phyla (Figure 7a). Attempts to align each of the A to F modules separately and enlarging the search to the $120 \mathrm{~kb}$ flanking Six3 in the Xenopus laevi, chicken, mouse, and human genomes were unsuccessful in detecting alignable sequences using the VISTA and multialign software [43,44]. Thus, only the $\mathrm{G}$ and L modules were highly conserved and similarly organized in all genomes, whereas the sequences that constitute the $\mathrm{H}$ and I modules in fishes were conserved but fragmented in a larger stretch of DNA in the other genomes analysed (Figure $7 \mathrm{~b}$ ), with the exception of the marsupial opossum, in which the I block was co-linear with that of fishes (data not shown). In spite of fragmentation, transgenic embryos, carrying the human sequence that included the $\mathrm{G}$ module and the dispersed $\mathrm{H}$ and I sequences (Figure 7c), exhibited spatio-temporal EGFP expression in the developing brain identical to that observed in the equivalent medaka genomic region (Figure $7 \mathrm{~d}-\mathrm{i}$ ). In addition, reporter expression was observed in the lens placode/vesicle. This suggested that although control of at least part of Six3 expression in the brain has been conserved, its regulation during lens development has undergone a reorganization of the appropriate cis-regulatory elements during evolution (data not shown).

Although the human construct (h-cI) we injected drove EGFP expression only in the late olSix3.2 expression domain, 
according to what expected given the sole presence of modules $\mathrm{G}$ to $\mathrm{L}$, we could not exclude that this human region contained regulatory information not readable in fish. Thus, to rule out possible cross-species interferences, we amplified from genomic DNA the equivalent Xenopus region, in which the $\mathrm{G}$ to L elements are organized as in humans (Figure $7 \mathrm{a}$ ). Transgenesis analysis in Xenopus embryos using a construct containing this fragment (X-cI; Figure $7 \mathrm{c}$ ) yielded results equivalent to those observed with the human fragment; EGFP reporter expression was detected only at later stages of brain development in the expected domain of Xsix3.2 expression (Figure $7 \mathrm{j}, \mathrm{k}$ ). This supports the idea that the regulatory information for early Six 3 expression in vertebrates other than fishes reside in as yet unidentified genomic regions.

\section{Discussion}

Six3 is an important regulator of vertebrate forebrain development. Gene regulatory network models predict that the precise spatio-temporal expression pattern of genes fundamental for embryo development must be orchestrated by the interaction of various regulatory regions [17]. Supporting the model, we functionally demonstrated that the entire expression of the newly identified olSix3.2 is orchestrated by the combined use of seven different cis-regulatory modules (Figure 6) and that at least part of this regulation is conserved in the Six3 locus of vertebrates other than fishes. Two main 'enhancer' modules (D and I) are responsible for olSix 3.2 expression at early and late stages of brain development, respectively. Their activity is spatially refined by the function of two 'silencers' and two 'silencer blockers'. In addition, olSix3.2 expression in the lens ectoderm and in the differentiating retina requires the combined activity of five different cis-regulatory modules. This apparently simple regulation may hide additional organization, as we have demonstrated for the I enhancer, in which an organized sequence of cis-regulatory elements control the posterior to anterior expression of olSix 3.2 in the brain.

The availability of different genome sequences and the development of analytical bioinformatic tools have facilitated study of cis regulation of a number of genes with evolutionary conserved roles in vertebrate embryonic development. Some of these studies have focused, as has ours, on a specific gene or a gene cluster, identifying enhancers that are involved in the control of specific expression domains [21,23,25,45-49]. However, possibly because of the size of the genomic regions that are involved, or to the laborious and time consuming use of mice, or the limitations of chick electroporation in validating regulatory activities, these studies have mostly focused on each enhancer as a separate entity, thus missing the effects of possible cooperative activities. Other recent and extremely informative studies, based on medium or small throughput screens in zebrafish, have instead systematically tested the autonomously enhancing function of large numbers of highly conserved noncoding elements positioned in areas surround- ing developmentally important genes, with positive identification only of a fraction of them [21,39]. Because each element is tested in an unconstrained context, negative regulators as well as modulatory functions of surrounding endogenous elements are also undetected using these approaches [39]. In contrast, possibly benefiting from the high transgenesis efficiency of the medaka fish [50] and its compact genome, we were able to assign enhancer, silencer, and modulatory functions to the majority of the highly conserved noncoding elements surrounding the Six 3 gene in fishes. Testing different combinations of these elements, we have also established their required interactions for proper expression of the gene. Thus, to our knowledge, we provide the first description of the regulatory code necessary for the expression of a vertebrate gene and offer a unique framework to define the entire interplay of trans-acting factors that control the evolutionary conserved use of Six 3 during forebrain development.

Teleosts are the most diverse class of vertebrates with a huge variety of different species; they are characterized by broad size range and dynamic organization of genomes, which are the result of an initial genome duplication followed by subsequent independent evolution of the different lineages [51,52]. Comparison of divergent teleost genomes largely separated in the phylogenetic tree, such as the medaka and zebrafish genomes (approximately 115 to 200 million years [28]), is thus a powerful tool with which to study gene regulatory mechanisms. Adopting this strategy, we identified a cluster of potential regulatory modules in the Six3 locus, which were barely identifiable in a comparison among mammalian genomes (compare Figure 2a with Figure 7a). VISTA analysis of the available genomic sequences flanking the homologous vertebrate Six 3 genes revealed several blocks of highly conserved noncoding sequences in the gene surroundings. Although a few of these blocks were located downstream of the coding sequence (data not shown), we demonstrated that the pattern of olSix3.2 expression could be recapitulated by $4.5 \mathrm{~kb}$ of genomic sequence flanking the 5 ' end of the gene. This conclusion is based on a relatively efficient (roughly $20 \%$ of injected embryos) and highly reproducible (basically 100\%, albeit with different EGFP intensity, thus excluding chromosomal position effects) transgenic analysis using three independent and stable medaka lines generated for all of the constructs we tested. Thus, we are fairly confident that we identified the main regulatory region for olSix3.2, although we cannot entirely exclude the possibility that additional or duplicated regulatory elements positioned in untested regions may contribute to a refinement of the main expression domain. Indeed, redundant cis-regulatory elements have been reported to control specific expression domains in different genes, including Otx2, Shh, and Sox2 [22,23,25].

According to our analysis, the regulatory region of olSix 3.2 is relatively compact as compared with those reported for other genes that are involved in neural development, such as Sox2, Sox9, Otx2, Pax6, and Shh, for which enhancers located in the 
(a)

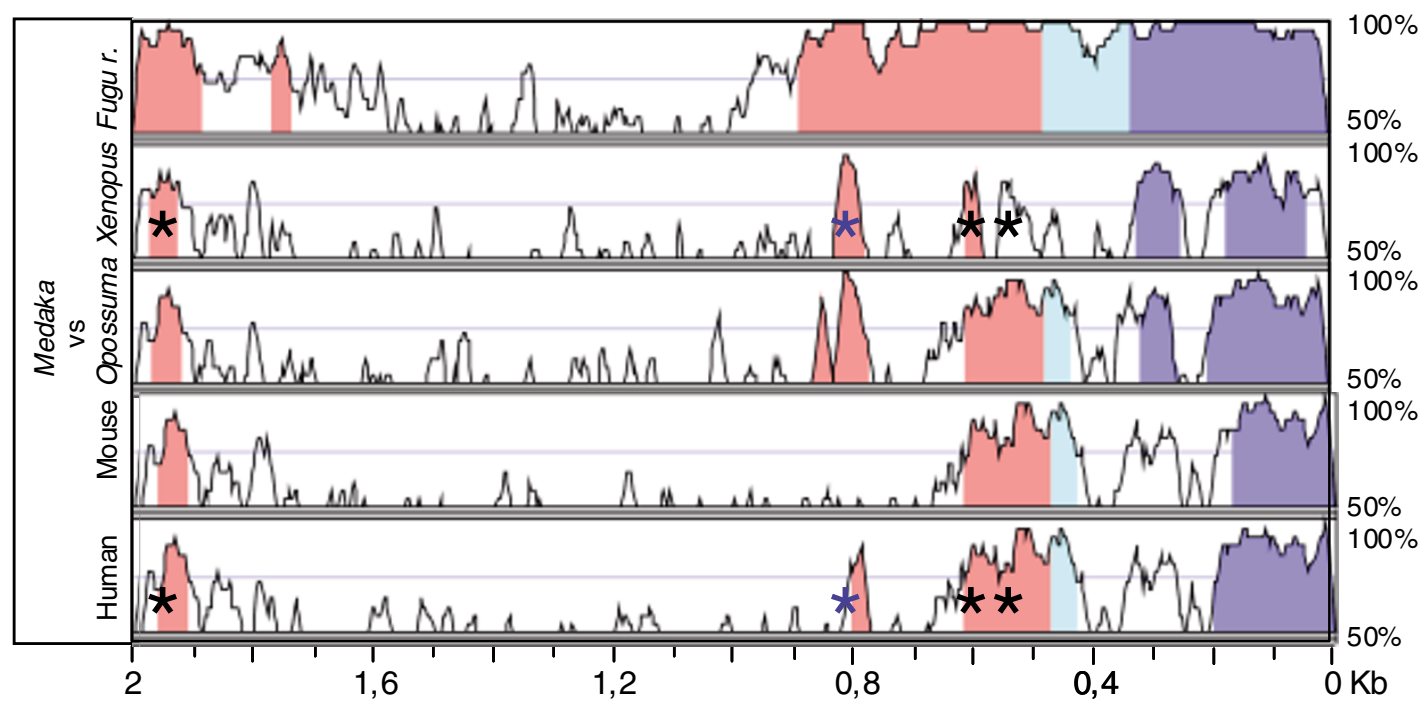

(b)

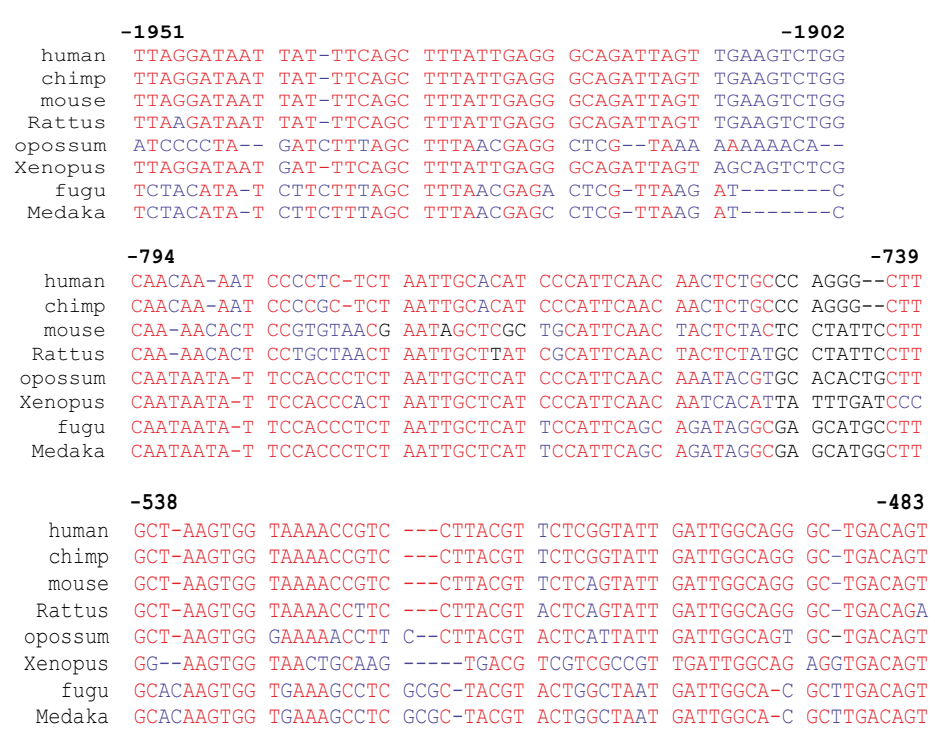

(c)
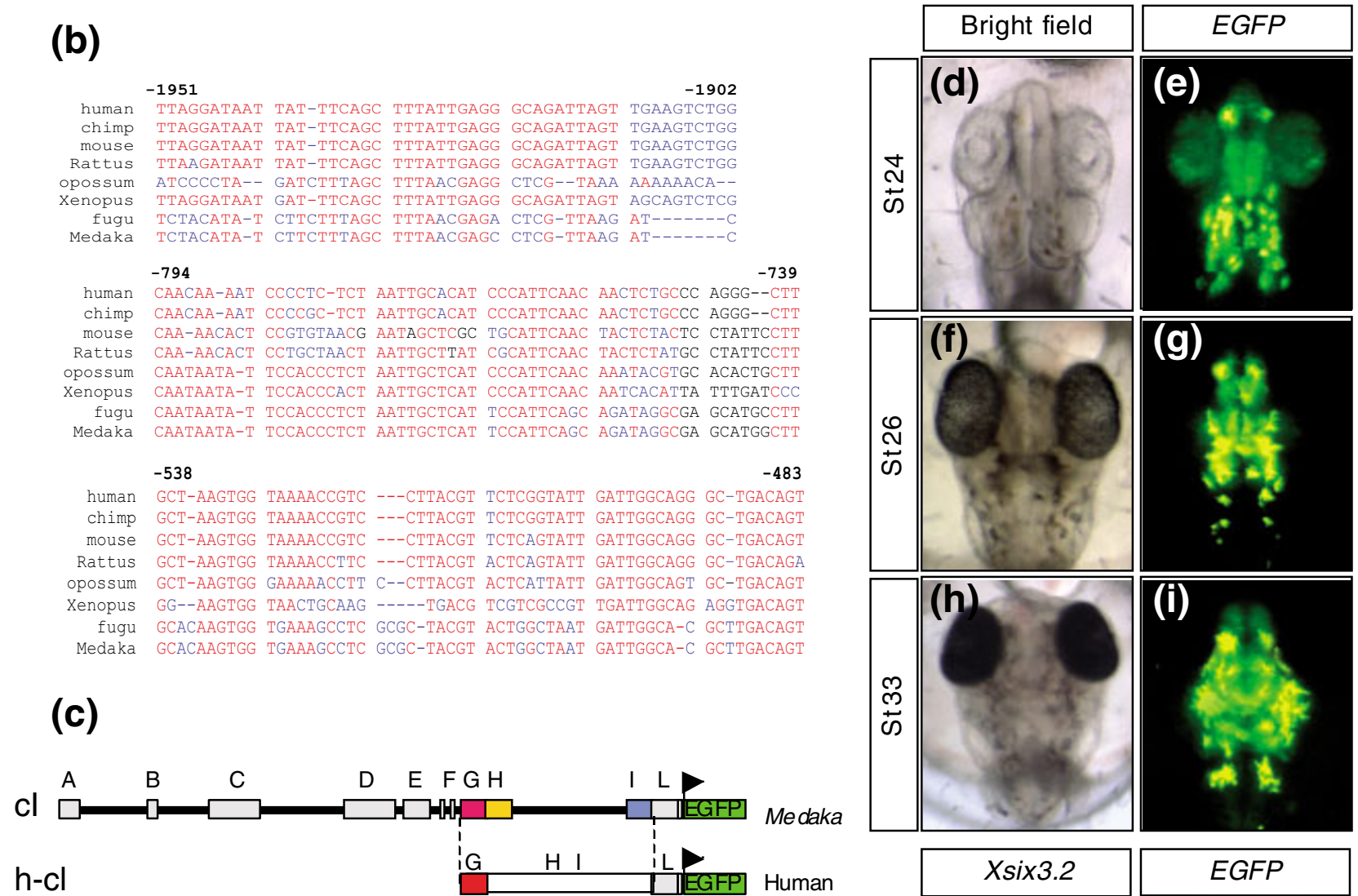

C TACTCTACTC СТATTCCTT

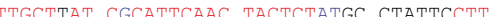

possum CAATAATA-T TCCACCCTCT AATTGCTCAT CCCATTCAAC AAATACGTGC ACACTGCTT

Xenopus CAATAATA-T ICCACCCACT AATTGCTCAT CCCATTCAAC AATCACATTA TTTGATCCC

$-538$

$-483$

human GCT-AAGTGG TAAAACCGTC ---CTTACGT TCTCGGTATT GATTGGCAGG GC-TGACAGT chimp GCT-AAGTGG TAAAACCGTC ---CTTACGT TCTCGGTATT GATTGGCAGG GC-TGACAGT TAACTGCAAG --.--TGACG TCGTCGCCGT TGATTGGCAG AGGTGACAGT Medaka GCACAAGTGG TGAAAGCCTC GCCC-TACGT
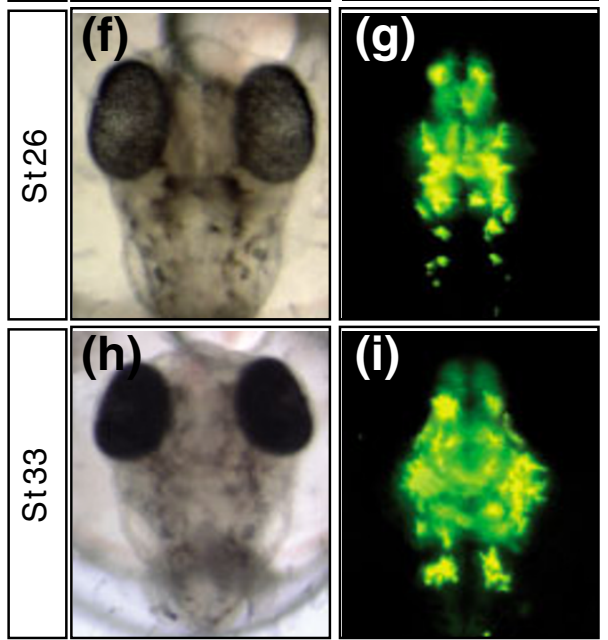

$\mathrm{h}-\mathrm{cl}$
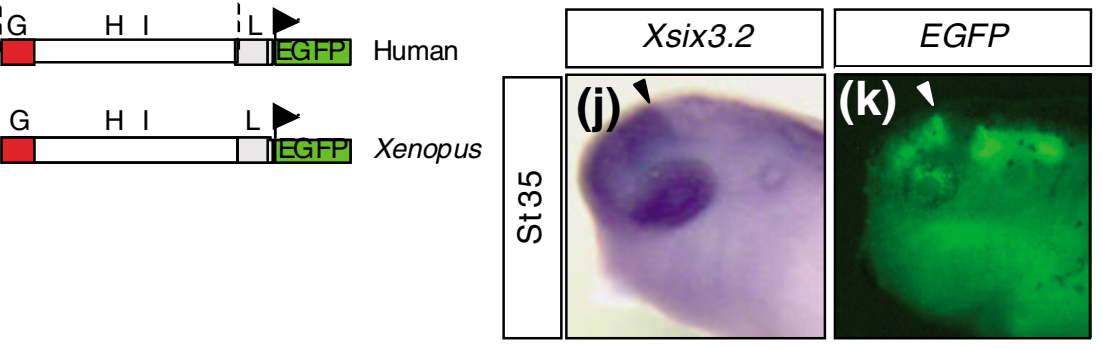

Figure 7 (see legend on next page) 
Figure 7 (see previous page)

Modules G, H, and I are functionally conserved in humans. (a) VISTA comparison (90\% identity over 25 base pairs) of the medaka olSix3.2 genomic region plotted against those of other vertebrates, as indicated. The analysis identifies highly conserved noncoding regions (pink peaks) corresponding to modules $\mathrm{G}$ (asterisk) and $\mathrm{L}$ (two asterisks), and to a partial I element (blue asterisk). The light and dark blue peaks correspond to the 5 '-untranslated region and coding sequence of Six3, respectively. (b) Nucleotide sequence alignment of module I from different vertebrates where partially or completely conserved sequences are indicated in blue or red, respectively. Nonconserved sequences are in black. The nucleotide positions are relative to the human genomic sequence. (c) Schematic representation of the human (h-cl) and Xenopus ( $\mathrm{X}-\mathrm{cl}$ ) constructs, containing the $\mathrm{G}$ (red box), $\mathrm{H}$, and $\mathrm{I}$ sequences, used to generate transient Xenopus and stable transgenic medaka lines. The mixed $\mathrm{H}$ and I sequences are represented as a striped blue and yellow box. (d, $\mathbf{f}$, and h) Bright field images and (e, g, and i) epi-fluorescence dorsal views of h-cl transgenic medaka embryos at different stages of development, as indicated in the panels. ( $\mathbf{j}$ and $\mathbf{k}$ ) Lateral views of St35 Xenopus embryos hybridized with a specific probe for Xsix3.2 or injected with X-cl. Note that in both Xenopus and medaka embryos reporter expression recapitulates Six 3 expression at the corresponding stages of development.

region of 10, 100, and even 1,000 kb away from their promoters have been reported [23-27,53,54], even in the compact Fugu genome [46]. Genes with complex patterns of expression are predicted to have more regulatory elements and occupy significantly more space in the genome than those with simpler expressions that are restricted to populations of cells with similarities or shared identity [55]. It is thus possible that the compactness of the olsix3.2 regulatory region might reflect the association that exists among the main territories in which the gene is expressed. Indeed, the specification of telencephalic and eye fields appears to be closely linked [2], and the initial expression of olSix3.2 in both regions appears to depend on the activity of a single enhancer element (D) and a distal silencer (A), which constrains the expression domain to the anteriormost neural tube. This hypothesis could also explain why the combined activities of five different modules ( $\mathrm{D}$ to $\mathrm{H}$ ) are instead needed to control expression in the lens placode, which is the only non-neural domain of olSix3.2 expression. Nevertheless, compactness does not appear to be, at least in this case, a reflection of simplicity, because each of the conserved modules may include additional regulatory organization. This is the case of module I, which is the main enhancer involved in the late embryonic expression of the gene. Stepwise and internal deletions of this module have revealed a peculiar organization, in a $5^{\prime}$ to $3^{\prime}$ direction, of a series of cis-regulatory elements that are required for the posterior to anterior spatio-temporal expression of olSix3.2 in the thalamus, hypothalamus, and telencephalon. The activity of the I module is refined by a silencer, $\mathrm{G}$, the activity of which is modulated by two silencer blockers that act in a temporal sequence, thus establishing an elaborate control code. Furthermore, although the L module per se has no activity, we cannot totally exclude the possibility that this module might contribute, together with modules $\mathrm{E}$ to $\mathrm{H}$, to the regulation of $\mathrm{I}$, because it was present in the constructs used for this analysis.

Alternatively, the short-range regulation of olSix3.2 may be linked to the chromosomal localization of the Six genes, which are organized in two evolutionarily conserved clusters [56]. Although the expression of the other Six family members (Six1, Six2, Six4, and Six 5 ) is mostly associated with tissues of mesodermal and ectodermal origin [3], it is possible that genes within the same cluster (Six4, Six1, and Six6) will share a few regulatory elements, which might have imposed constrains against rearrangement during evolution [57].

In silico comparison identified ten conserved modules in the teleost Six3 locus. Transgenic analysis in medaka demonstrated clear regulatory activity for seven of them, whereas modules B, C, and L did not influence EGFP reporter expression. Although these modules might have subtle regulatory activities below the resolution of our analysis, their conservation could reflect other important roles in gene transcription control, such as regulation of chromatin structure or - in the particular case of module L - they may contribute to minimal promoter functions.

The regulatory region we have studied belongs to a newly identified medaka Six 3 gene, namely olSix3.2. Genomic organization and phylogenetic analysis suggests that olSix 3.2 is more closely related to the mammalian Six 3 than the previously identified olSix3.1 [12]. Like its mammalian homolog [4], olSix3.2 is strongly expressed in various forebrain regions where its paralog is not expressed. Our comparative expression study suggests that the combination of expression domains of olSix3.1, olSix3.2, and the related olSix6 correspond to the combined tissue distribution observed for the mouse and chick Six 3 and Six6 [32-34], with a preponderant expression of olSix3.1 in the eye, of olSix3.2 in the telencephalic and thalamic regions, and of olSix 6 in the hypothalamus. Genetic abrogation studies in mice demonstrated that Six 3 is necessary for the formation of forebrain, which is absent in homozygous embryos [5]. Genetic deletion of Six6 instead is associated with pituitary defects, absence or hypoplasia of the optic nerves, and chiasm and alteration in neural retina proliferation [58]. How the functions of olSix3.1, olSix3.2, and olSix6 relate to those described in the mouse for Six3 and Six6 is still unresolved and knock-down analysis of all three genes in medaka will be necessary to address this issue. Thus far, morpholino-based knock-down of olSix3.1 results in forebrain and eye defects, including loss of optic stalk markers [9], whereas preliminary analysis indicates that olSix3.2 morphants are characterized by strong ventral forebrain defects with minor eye malformations (De la Torre A, Conte I, Bovolenta P, unpublished observations), suggesting that the two olSix 3 paralogs may cover Six 3 as well as part of the mouse Six 6 functions, a possibility that is also supported 
by the phylogenetic position of olSix3.1, which falls almost in between the Six 3 and Six6 branches of the Six gene family (Additional data file 2).

Comparative analysis of the regulatory code of the three medaka genes currently ongoing in our laboratory might be useful in complementing these studies by providing insights into the sub-functionalization or neo-functionalization of olSix3.1, olSix3.2, and olSix6 as compared with their mammalian counterparts. Furthermore, they will help to elucidate whether Six 3 and Six6 have arisen from the duplication of a common ancestor, as previously proposed [56], possibly duplicating at least part of their regulatory region. This is an important point because, with the comparison parameters used, we were unable to identify in other vertebrate species the conservation and distribution of the A to $\mathrm{F}$ regulatory modules characterized in fishes. This is particularly important for the A and D modules, which are the main regulators of early Six 3 expression in fishes. Informatics searches of corresponding regions in mammalian genomes yielded no clear information, suggesting that these modules might be present outside the regions that we analyzed or they might have evolved differently in other vertebrate genomes, making their search even more difficult than that of the $\mathrm{H}$ and I modules. Alternatively, these modules may represent a new acquisition of olSix3.2 caused by teleost genome duplication.

In our study, we demonstrated strong functional conservation between fishes and other vertebrates only for the $\mathrm{G}, \mathrm{H}$, and I modules, which control late expression of olSix3.2. The sequences that compose the $\mathrm{H}$ and I modules in fishes were intermixed and differently arranged in other vertebrate genomes, although their function was strongly conserved when assayed in medaka and Xenopus transgenesis. This suggests that sequences from different vertebrates are activated by common transcription factors, although the binding sites for these factors might be distributed, oriented, or represented in different numbers among species. An additional explanation for the different arrangement of the $\mathrm{H}$ and I modules might be species-specific nucleotide modifications, which have been proposed to contribute to gene transcriptional evolution [59-61].

Conservation of regulatory function between human and fish in the absence of clear sequence conservation has previously been reported also for the RET gene. In this case, lack of correlation between the two events was even more marked, and different in silico analysis designed to detect shorter stretches of sequence similarities or the existence of inversion and rearrangement failed to detect alignable sequences [62]. Thus, our data, together with few additional observations [63-65], strongly support the idea proposed by Fisher and colleagues [62] that some relevant regulatory information might be conserved among species at a level that is not detectable using genomic sequence alignment.

\section{Conclusion}

Our study established the cis-regulatory code required for the proper expression of olSix3.2 and demonstrates that there is a need to test different combinations of highly conserved putative cis-regulatory regions to elucidate how each conserved element contributes to the spatio-temporal control of gene expression. In fact, one limitation of previous studies that have used transgenic analysis to test the function of highly conserved noncoding sequences is the identification of single enhancers uprooted from possible interactions with the remaining regulatory elements. Our comprehensive description of the olSix 3.2 regulatory code is now a powerful starting point from which to define the entire interplay of trans-acting factors that control the evolutionarily conserved use of Six 3 during forebrain development. From a broader perspective, this type of information will be necessary to elucidate the composition and evolution of vertebrate gene regulatory networks, as compared with those of invertebrates such as Drosophila and sea urchin, in which this type of information is accumulating at a much faster pace [17].

\section{Materials and methods \\ Microinjection and establishment of transgenic lines}

Adult and embryonic medaka fishes (Oryzia latipes) from the Cab inbred strain were used throughout the study. Fertilized eggs were collected immediately and incubated at 4 to $10^{\circ} \mathrm{C}$ in Yamamoto's embryo rearing medium to suppress further development [66]. DNA was prepared using a High Pure Plasmid Isolation Kit (Roche, Basel, Switzerland). DNA injections (10 $\mathrm{ng} / \mu \mathrm{l}$ DNA in ISceI enzyme reaction) were performed as previously described [50]. Embryos were staged according to the method proposed by Iwamatsu [66], raised to sexual maturity, and transgenic founder fishes were identified by out-crossing to wild-type fishes. Transcriptional activation of the constructs was monitored by EGFP expression observed in living embryos under UV fluorescent stereo-microscopy (Leica Microsystems, Wetzlar, Germany). Xenopus laevis embryos were obtained and raised as described previously [21]. Xenopus transgenesis was performed following the same procedures as used for the medaka embryos.

\section{Whole-mount in situ hybridization}

Whole-mount in situ hybridizations were performed as previously described using digoxigenin labelled riboprobes [29]. Anti-sense and sense riboprobes for medaka olSix3.1, olSix3.2, and olSix6 and the Xenopus Xsix3.2 were used. A minimum of 40 embryos were hybridized for each marker and condition. In toto hybridized embryos were photographed, embedded in gelatine/albumine block, and further sectioned using a vibratome (Leica Microsystems, Wetzlar, Germany).

\section{Sequence analysis}

The vertebrate Six 3 genomic sequences were retrieved from public databases: Genome Browser UCSC [67] and JGI [68]. 
The genomic sequence of olSix 3.2 was isolated from medaka genomic DNA using the following primers: olSix 3 forward CCTCATTAAATGTCGCTAAC, and olsix3 reverse cgcctaatgacac cagcctc. Sequence alignments were performed using the VISTA [43] and Multalign programs [44], which are available at the corresponding websites $[69,70]$. The criterion used for comparisons was a minimum $75 \%$ nucleotide identity with a window size of over $100 \mathrm{bp}$. Phylogenetic analysis was performed using the PHYLIP package [71]. The results were plotted using the Tree-view software package [72]. olSix3.2 protein sequences were scanned for motifs using online software available at HGMP [73] and NCBI [74].

\section{Isolation of olSix3.2 cDNA}

Total RNAs from medaka embryos at different stages were isolated by RNAzol B (Campro Scientific, Berlin, Germany) and treated with Dnase I (Invitrogen, Carlsbad, CA). RT-PCR reactions were performed using SUPERSCRIPT II (Invitrogen, Carlsbad, CA), as described previously [75]. PCR using olSix3.2 specific primers was performed using $2 \mu \mathrm{l}$ of the reverse transcription reaction as a template with the High Fidelity PCR system (Roche, Basel, Switzerland). Oligonucleotide primers used to isolate olSix3.2 cDNA are listed in Additional data file 4 .

\section{Plasmid constructions}

A $4.5 \mathrm{~kb}$ region of olSix 3.2 genomic sequence containing nine nucleotides (corresponding to the first three amino acids) of the coding region was cloned in frame with EGFP reporter gene into the pSKII-ISceI-EGFP vector [50], to create the cI construct. Xenopus and human sequences were amplified from corresponding genomic DNA and cloned in the pSKIIISceI-EGFP vector with the same strategy. The medaka

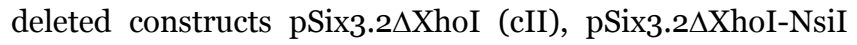
(cVIII), and pSix3.2 $\Delta$ XbaI-HindIII (cIX) were obtained by digesting the pSix3.2-4.5 $\mathrm{kb}$ construct using the indicated enzymes. All the other deleted constructs (pSix3.2 $\Delta$ el1, pSix3.2 $\Delta$ el2, pSix3.2 $\Delta$ el $3, \quad$ pSix3.2 $\Delta$ el $4, \quad$ pSix3.2 $\Delta$ el 5 , pSix3.2 $\Delta$ el6, and pSix3.2 $\Delta$ el7; cXXIII to cXXIX) were obtained by PCR amplification from pSix3.2-4.5 kb and then cloned into pSKII-ISceI-EGFP vector. The A, B, and C modules were deleted by restriction enzyme digestion (A, NarI/ KpnI; B, BtsI/BglII; and C, BamHI/ClaI) and inserted (in sense and anti-sense orientations) into the polylinker of

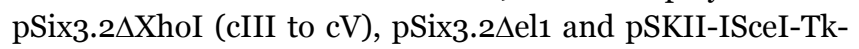
EGFP (containing the tyrosine kinase minimal promoter) vectors to test their potential regulatory activity. All of the other modules were amplified and cloned (in sense and antisense orientations) into the polylinker of pSix3.2 $\Delta$ el1 (cVI to cVII, cX, and cXII to cXVI) and pSKII-ISceI-Tk-EGFP (cXI and $\mathrm{cXVII}$ to $\mathrm{cXXII}$ ). The primer sequences used to generate these constructs are shown in Supplementary Table I. All constructs were verified by automated sequencing.

\section{Additional data files}

The following additional data are available with the online version of this manuscript. Additional data file 1 is a Figure reporting the amino acid sequence alignment of Six3 genes from different vertebrate species. Additional data file 2 is a figure illustrating the phylogenetic tree of the SIX family. Additional data file 3 provides the precise nucleotide sequences of modules A to $\mathrm{L}$ described in the report. Additional data file 4 is a table listing the sequences of the primers used to amplify the DNA fragments, which were used to design the different constructs described in the report.

\section{Acknowledgements}

We are grateful to Drs WA Harris, $\mathrm{H}$ Kondoh, and M Manzanares for critical reading of the manuscript, and to Dr J Wittbrodt and members of our laboratory for many helpful suggestions. We wish to thank I Dompablo for excellent technical assistance. This study was supported by grants from Spanish Ministerio de Educación y Ciencia (BFU-2004-0I585) and in part by the EU (QLG3-CT-200I-0I460) and the HFSPO (RGP0040/200I-M) to PB. A Telethon Foundation (GFP03007) and MEC (SB2003-0182) fellowships supported the postdoctoral work of IC.

\section{References}

I. Wilson SW, Houart C: Early steps in the development of the forebrain. Dev Cell 2004, 6:167-181.

2. Esteve $P$, Bovolenta $P$ : Secreted inducers in vertebrate eye development: more functions for old morphogens. Curr Opin Neurobiol 2006, 16:13-19.

3. Rodríguez de Córdoba S, Gallardo ME, Lopez-Rios J, Bovolenta P: The human Six family of homeobox genes. Curr Genomics 200 I, 2:23I-242.

4. Conte I, Morcillo J, Bovolenta P: Comparative analysis of Six 3 and Six 6 distribution in the developing and adult mouse brain. Dev Dyn 2005, 234:718-725.

5. Lagutin OV, Zhu CC, Kobayashi D, Topczewski J, Shimamura K Puelles L, Russell HR, McKinnon PJ, Solnica-Krezel L, Oliver G: Six3 repression of Wnt signaling in the anterior neuroectoderm is essential for vertebrate forebrain development. Genes Dev 2003, 17:368-379.

6. Wallis DE, Muenke M: Molecular mechanisms of holoprosencephaly. Mol Genet Metab 1999, 68:I26-I38.

7. Pasquier L, Dubourg C, Gonzales M, Lazaro L, David V, Odent S, Encha-Razavi F: First occurrence of aprosencephaly/atelencephaly and holoprosencephaly in a family with a SIX3 gene mutation and phenotype/genotype correlation in our series of SIX3 mutations. J Med Genet 2005, 42:e4.

8. Liu W, Lagutin OV, Mende M, Streit A, Oliver G: Six3 activation of Pax6 expression is essential for mammalian lens induction and specification. EMBO / 2006, 25:5383-5395.

9. Carl M, Loosli F, Wittbrodt J: Six3 inactivation reveals its essential role for the formation and patterning of the vertebrate eye. Development 2002, 129:4057-4063.

10. Bernier G, Panitz F, Zhou X, Hollemann T, Gruss P, Pieler T: Expanded retina territory by midbrain transformation upon overexpression of Six6 (Optx2) in Xenopus embryos. Mech Dev 2000, 93:59-69.

II. Kobayashi M, Nishikawa K, Suzuki T, Yamamoto M: The homeobox protein Six 3 interacts with the Groucho corepressor and acts as a transcriptional repressor in eye and forebrain formation. Dev Biol 200I, 232:3I5-326.

12. Loosli F, Winkler S, Wittbrodt J: Six3 overexpression initiates the formation of ectopic retina. Genes Dev 1999, 1 3:649-654.

13. Lopez-Rios J, Tessmar K, Loosli F, Wittbrodt J, Bovolenta P: Six 3 and Six 6 activity is modulated by members of the groucho family. Development 2003, I30:185-195.

14. Zhu CC, Dyer MA, Uchikawa M, Kondoh H, Lagutin OV, Oliver G: Six3-mediated auto repression and eye development requires its interaction with members of the Grouchorelated family of co-repressors. Development 2002, 
| 29:2835-2849.

15. Zuber ME, Perron M, Philpott A, Bang A, Harris WA: Giant eyes in Xenopus laevis by overexpression of XOptx2. Cell 1999, 98:34I-352.

16. Del Bene F, Tessmar-Raible K, Wittbrodt J: Direct interaction of geminin and Six3 in eye development. Nature 2004, 427:745-749.

17. Davidson EH, Erwin DH: Gene regulatory networks and the evolution of animal body plans. Science 2006, 3 I I :796-800

18. Levine $M$, Davidson $E H$ : Gene regulatory networks for development. Proc Natl Acad Sci USA 2005, 102:4936-4942.

19. Michelson AM: Deciphering genetic regulatory codes: a challenge for functional genomics. Proc Natl Acad Sci USA 2002, 99:546-548.

20. Allende ML, Manzanares M, Tena JJ, Feijoo CG, Gomez-Skarmeta JL: Cracking the genome's second code: enhancer detection by combined phylogenetic footprinting and transgenic fish and frog embryos. Methods 2006, 39:2I2-219.

21. de la Calle-Mustienes E, Feijoo CG, Manzanares M, Tena JJ, Rodriguez-Seguel E, Letizia A, Allende ML, Gomez-Skarmeta JL: A functional survey of the enhancer activity of conserved noncoding sequences from vertebrate Iroquois cluster gene deserts. Genome Res 2005, I5:1061-1072.

22. Jeong Y, El-Jaick K, Roessler E, Muenke M, Epstein DJ: A functional screen for sonic hedgehog regulatory elements across a I Mb interval identifies long-range ventral forebrain enhancers. Development 2006, 133:76|-772.

23. Kurokawa D, Kiyonari H, Nakayama R, Kimura-Yoshida C, Matsuo I, Aizawa S: Regulation of Otx 2 expression and its functions in mouse forebrain and midbrain. Development 2004, | $31: 3319-3331$.

24. Kurokawa D, Takasaki N, Kiyonari H, Nakayama R, Kimura-Yoshida C, Matsuo I, Aizawa S: Regulation of Otx2 expression and its functions in mouse epiblast and anterior neuroectoderm. Development 2004, I3 I:3307-33 I7

25. Uchikawa M, Ishida $Y$, Takemoto $T$, Kamachi $Y$, Kondoh H: Functional analysis of chicken Sox 2 enhancers highlights an array of diverse regulatory elements that are conserved in mammals. Dev Cell 2003, 4:509-5I9.

26. Bagheri-Fam S, Barrionuevo F, Dohrmann U, Gunther T, Schule R, Kemler R, Mallo M, Kanzler B, Scherer G: Long-range upstream and downstream enhancers control distinct subsets of the complex spatiotemporal Sox9 expression pattern. Dev Biol 2006, 29 I:382-397

27. Bagheri-Fam S, Ferraz C, Demaille J, Scherer G, Pfeifer D: Comparative genomics of the SOX9 region in human and Fugu rubripes: conservation of short regulatory sequence elements within large intergenic regions. Genomics 200I, 78:73-82.

28. Wittbrodt J, Shima A, Schartl M: Medaka: a model organism from the far East. Nat Rev Genet 2002, 3:53-64.

29. Loosli F, Koster RW, Carl M, Krone A, Wittbrodt J: Six3, a medaka homologue of the Drosophila homeobox gene sine oculis is expressed in the anterior embryonic shield and the developing eye. Mech Dev 1998, 74:159-164.

30. Ghanbari H, Seo HC, Fiose A, Brandli AW: Molecular cloning and embryonic expression of Xenopus Six homeobox genes. Mech Dev 200I, 10I:27I-277.

3I. Seo HC, Drivenes, Ellingsen S, Fjose A: Expression of two zebrafish homologues of the murine Six 3 gene demarcates the initial eye primordia. Mech Dev 1998, 73:45-57.

32. Bovolenta P, Mallamaci A, Puelles L, Boncinelli E: Expression pattern of cSix3, a member of the Six/sine oculis family of transcription factors. Mech Dev 1998, 70:20I-203.

33. Oliver G, Mailhos A, Wehr R, Copeland NG, Jenkins NA, Gruss P: Six 3, a murine homologue of the sine oculis gene, demarcates the most anterior border of the developing neural plate and is expressed during eye development. Development 1995, I 2 I:4045-4055.

34. Lopez-Rios J, Gallardo ME, Rodriguez de Cordoba S, Bovolenta P: Six9 (Optx2), a new member of the six gene family of transcription factors, is expressed at early stages of vertebrate ocular and pituitary development. Mech Dev 1999, 83:155-159.

35. Toy J, Yang JM, Leppert GS, Sundin OH: The optx2 homeobox gene is expressed in early precursors of the eye and activates retina-specific genes. Proc Natl Acad Sci USA 1998, 95:10643-10648.

36. Hardison RC: Conserved noncoding sequences are reliable guides to regulatory elements. Trends Genet 2000, 16:369-372.

37. Thomas JW, Touchman JW, Blakesley RW, Bouffard GG, BeckstromSternberg SM, Margulies EH, Blanchette M, Siepel AC, Thomas PJ, McDowell JC, et al:: Comparative analyses of multi-species sequences from targeted genomic regions. Nature 2003, 424:788-793.

38. Nobrega MA, Pennacchio LA: Comparative genomic analysis as a tool for biological discovery. J Physiol 2004, 554:3I-39.

39. Woolfe A, Goodson M, Goode DK, Snell P, McEwen GK, Vavouri T, Smith SF, North P, Callaway H, Kelly K, et al:: Highly conserved non-coding sequences are associated with vertebrate development. PLoS Biol 2005, 3:e7.

40. Alfano G, Vitiello C, Caccioppoli C, Caramico T, Carola A, Szego MJ, Mclnnes RR, Auricchio A, Banfi S: Natural antisense transcripts associated with genes involved in eye development. Hum $\mathrm{Mol}$ Genet 2005, 14:913-923.

4I. Oki M, Kamakaka RT: Blockers and barriers to transcription: competing activities? Curr Opin Cell Biol 2002, 14:299-304.

42. West AG, Fraser P: Remote control of gene transcription. Hum Mol Genet 2005, I4(Spec No I):RIOI-RIII.

43. Frazer KA, Pachter L, Poliakov A, Rubin EM, Dubchak I: VISTA: computational tools for comparative genomics. Nucleic Acids Res 2004:W273-W279.

44. Corpet F: Multiple sequence alignment with hierarchical clustering. Nucleic Acids Res 1988, 16:1088I-10890

45. Kimura C. Takeda N, Suzuki M, Oshimura M, Aizawa S, Matsuo I: Cisacting elements conserved between mouse and pufferfish Otx2 genes govern the expression in mesencephalic neural crest cells. Development 1997, 124:3929-3941.

46. Kimura-Yoshida C, Kitajima K, Oda-Ishii I, Tian E, Suzuki M, Yamamoto M, Suzuki T, Kobayashi M, Aizawa S, Matsuo I: Characterization of the pufferfish Otx2 cis-regulators reveals evolutionarily conserved genetic mechanisms for vertebrate head specification. Development 2004, I3 1:57-7I.

47. Takemoto T, Uchikawa M, Kamachi Y, Kondoh H: Convergence of Wnt and FGF signals in the genesis of posterior neural plate through activation of the Sox 2 enhancer $\mathbf{N}-\mathrm{I}$. Development 2006, 133:297-306.

48. Hutcheson DA, Hanson MI, Moore KB, Le TT, Brown NL, Vetter ML: bHLH-dependent and -independent modes of Ath5 gene regulation during retinal development. Development 2005, 132:829-839.

49. Lee AP, Koh EG, Tay A, Brenner S, Venkatesh B: Highly conserved syntenic blocks at the vertebrate Hox loci and conserved regulatory elements within and outside Hox gene clusters. Proc Natl Acad Sci USA 2006, 103:6994-6999.

50. Thermes V, Grabher C, Ristoratore F, Bourrat F, Choulika A, Wittbrodt J, Joly JS: I-Scel meganuclease mediates highly efficient transgenesis in fish. Mech Dev 2002, I I 8:91-98.

5I. Furutani-Seiki M, Wittbrodt J: Medaka and zebrafish, an evolutionary twin study. Mech Dev 2004, I 2 I:629-637.

52. Muller F, Blader P, Strahle U: Search for enhancers: teleost models in comparative genomic and transgenic analysis of cis regulatory elements. Bioessays 2002, 24:564-572.

53. Kleinjan DA, Seawright A, Mella S, Carr CB, Tyas DA, Simpson TI, Mason JO, Price DJ, van Heyningen V: Long-range downstream enhancers are essential for Pax6 expression. Dev Biol 2006 299:563-581.

54. Lettice LA, Heaney SJ, Purdie LA, Li L, de Beer P, Oostra BA, Goode $D$, Elgar G, Hill RE, de Graaff E: A long-range Shh enhancer regulates expression in the developing limb and fin and is associated with preaxial polydactyly. Hum Mol Genet 2003, I 2:1725-1735.

55. Nelson CE, Hersh BM, Carroll SB: The regulatory content of intergenic DNA shapes genome architecture. Genome Biol 2004, 5:R25

56. Gallardo ME, Lopez-Rios J, Fernaud-Espinosa I, Granadino B, Sanz R, Ramos C, Ayuso C, Seller MJ, Brunner HG, Bovolenta P, et al: Genomic cloning and characterization of the human homeobox gene SIX6 reveals a cluster of SIX genes in chromosome 14 and associates SIX6 hemizygosity with bilateral anophthalmia and pituitary anomalies. Genomics 1999, 61:82-91.

57. Duboule D, Wilkins AS: The evolution of 'bricolage'. Trends Genet 1998, I 4:54-59.

58. Li X, Perissi V, Liu F, Rose DW, Rosenfeld MG: Tissue-specific regulation of retinal and pituitary precursor cell proliferation. Science 2002, 297: I I80-I I83.

59. Cowles CR, Hirschhorn JN, Altshuler D, Lander ES: Detection of 
regulatory variation in mouse genes. Nat Genet 2002, 32:432-437.

60. Wray GA: Transcriptional regulation and the evolution of development. Int J Dev Biol 2003, 47:675-684.

61. Teichmann SA, Babu MM: Gene regulatory network growth by duplication. Nat Genet 2004, 36:492-496.

62. Fisher S, Grice EA, Vinton RM, Bessling SL, McCallion AS: Conservation of RET regulatory function from human to zebrafish without sequence similarity. Science 2006, 3 I 2:276-279.

63. Oda-Ishii I, Bertrand V, Matsuo I, Lemaire P, Saiga H: Making very similar embryos with divergent genomes: conservation of regulatory mechanisms of Otx between the ascidians Halocynthia roretzi and Ciona intestinalis. Development 2005, I32:1663-1674.

64. Pennacchio LA, Rubin EM: Genomic strategies to identify mammalian regulatory sequences. Nat Rev Genet 200I, 2:100-109.

65. Gottgens B, Barton LM, Gilbert JG, Bench AJ, Sanchez MJ, Bahn S, Mistry S, Grafham D, McMurray A, Vaudin M, et al.: Analysis of vertebrate SCL loci identifies conserved enhancers. Nat Biotechnol 2000, 18:181-186.

66. Iwamatsu T: Stages of normal development in the medaka Oryzias latipes. Mech Dev 2004, I 2 I:605-6 I8.

67. UCSC Genome Bioinformatics [http://genome.ucsc.edu]

68. Eukaryotic Genomics [http://genome.jgi-psf.org]

69. VISTA [http://genome.lbl.gov/vista/index.shtml]

70. MultAlin [http://bioinfo.genopole-toulouse.prd.fr/multalin/multa lin.html]

7I. Retief JD: Phylogenetic analysis using PHYLIP. Methods Mol Biol 2000, I 32:243-258.

72. Page RD: TreeView: an application to display phylogenetic trees on personal computers. Comput Appl Biosci 1996, I 2:357-358.

73. HGMP/RFCGR [http://fugu.biology.qmul.ac.uk]

74. BLAST [http://www.ncbi.nlm.nih.gov/BLAST]

75. Conte I, Lestingi M, den Hollander A, Alfano G, Ziviello C, Pugliese M, Circolo D, Caccioppoli C, Ciccodicola A, Banfi S: Identification and characterisation of the retinitis pigmentosa I-like I gene (RPILI): a novel candidate for retinal degenerations. Eur J Hum Genet 2003, I I: 155-162. 